\title{
A Row-Column-Addressed 2D Probe with an Integrated Compound Diverging Lens
}

Engholm, Mathias; Beers, Christopher; Havreland, Andreas Spandet; Tomov, Borislav Gueorguiev; Jensen, Jørgen Arendt; Thomsen, Erik Vilain

Published in:

2018 IEEE International Ultrasonics Symposium (IUS)

Link to article, DOI:

10.1109/ULTSYM.2018.8579955

Publication date:

2018

Document Version

Peer reviewed version

Link back to DTU Orbit

Citation (APA):

Engholm, M., Beers, C., Havreland, A. S., Tomov, B. G., Jensen, J. A., \& Thomsen, E. V. (2018). A

Row-Column-Addressed 2D Probe with an Integrated Compound Diverging Lens. In 2018 IEEE International Ultrasonics Symposium (IUS) IEEE. https://doi.org/10.1109/ULTSYM.2018.8579955

\section{General rights}

Copyright and moral rights for the publications made accessible in the public portal are retained by the authors and/or other copyright owners and it is a condition of accessing publications that users recognise and abide by the legal requirements associated with these rights.

- Users may download and print one copy of any publication from the public portal for the purpose of private study or research.

- You may not further distribute the material or use it for any profit-making activity or commercial gain

- You may freely distribute the URL identifying the publication in the public portal 


\title{
A Row-Column-Addressed 2D Probe with an Integrated Compound Diverging Lens
}

\author{
Mathias Engholm*, Christopher Beers ${ }^{\dagger}$, Andreas Spandet Havreland*, Borislav Gueorguiev Tomov ${ }^{\ddagger}$, \\ Jørgen Arendt Jensen ${ }^{\ddagger}$, and Erik Vilain Thomsen* \\ * Department of Micro and Nanotechnology, Technical University of Denmark, DK-2800 Kgs. Lyngby, Denmark \\ ${ }^{\dagger} \mathrm{BK}$ Medical, PA-16803, USA. \\ ${ }_{\ddagger}$ Center for Fast Ultrasound Imaging, Department of Electrical Engineering, Technical University of Denmark, \\ DK-2800 Kgs. Lyngby, Denmark
}

\begin{abstract}
Planar 2D row-column-addressed (RCA) arrays can be an attractive alternative to fully-populated arrays due to their significantly lower channel count. However, these arrays can only look straight forward, which limits their utility. One way to increase their field of view is by applying a diverging lens. However, when common lens materials are used for a singlelayer diverging lens, they exhibit deficiencies in performance or form factor. A compound lens solution was integrated into a fully functioning probe to achieve a $30^{\circ}$ field-of-view (FOV) while retaining clinically-acceptable patient contact characteristics. The compound lens was fabricated of a $\mathrm{Bi}_{2} \mathrm{O}_{3}$ loaded RTV and an urethane, Hapflex 541.

Two similar developed probes were compared one with lens and one without. A curvilinear FOV of $28.5^{\circ}$ was obtained, which was slightly lower than the designed and was caused by small deformation of the lens during assembly. The output pressure was lowered a factor 6 and the center frequency decreased from $8.5 \mathrm{MHz}$ to $4.9 \mathrm{MHz}$ due to the lens. This was caused by the lens thickness, resulting in an increased attenuation of the transmitted signal. The difference between the two $\mathrm{dB}$ compressed frequency responses was observed to follow a linear tendency with a fitted slope of $-4 \mathrm{~dB} / \mathrm{MHz}$, which was in agreement with the estimated attenuation of the lens.
\end{abstract}

\section{INTRODUCTION}

Planar 2D row-column-addressed (RCA) 2D arrays can be an attractive alternative to fully-populated matrix arrays, as they offer volumetric imaging with a greatly reduced number of electrical connections [1]-[4]. However, an inherent problem of such arrays is the field-of-view (FOV), which is limited to the forward looking rectilinear volume region in front of the transducer. For applications such as abdominal and cardiac imaging, a curvilinear volume region is necessary.

By using a diverging lens in front of the RCA transducer, the inherent rectilinear $3 \mathrm{D}$ imaging FOV can be extended to a curvilinear volume region [5], [6]. Joyce and Lockwood presented a diverging lens, cast onto the face of a PZT transducer, made of RTV-11. The lens material and curvature was optimized using PZFlex/OnScale (OnScale, California, USA) and the resulting lens obtained a FOV of $45^{\circ}$ [5]. Bouzari et al. presented two concave lenses made of room temperature vulcanizing (RTV) rubber fabricated with two different radii, and it was shown that a FOV of $44.4^{\circ}$ could be obtained [7]. The disadvantage was that the lens was formed in RTV rubber, which has a sound velocity lower than tissue. The lens shape therefore has to be concave. A concave front curvature make patient contact very difficult, so a flat or convex front is usually a requirement, as air can be trapped between the patient and the transducer. Using a lens material with a sound velocity faster than tissue can solve this problem. The concern is that the center of the acoustic wave is attenuated more than the edge, resulting in worsened acoustic wave characteristics. Another concern is that faster materials often are stiffer, which can potentially be a problem for capacitive micromachined ultrasonic transducers (CMUTs), as this can influence the plate behavior. Another solution could be to use a compound lens of two or more materials to obtain a flat or convex front.

Such a diverging compound lens was designed and evaluated by Yang. et al. for photoacoustic computed tomography [8]. The aim was to increase the receive directivity of the detectors for photoacoustic computed tomography to avoid deformation of off-center targets. They utilized a concave shaped polydimethylsiloxane (PDMS) as one layer, and a convex shaped epoxy as the other. The $-6 \mathrm{~dB}$ acceptance angle was increased from $11^{\circ}$ to $55^{\circ}$.

Other compound lenses have been investigated for 1D transducers for elevation focusing, when a high mechanical strength or hardness are required to protect the acoustic stack, to protect the sole against cuts that can arise during surgical procedures, or if an impervious material is needed to make it easy to sterilize without compromising the array performance [9]-[11]. Such materials usually have a higher sound velocity than tissue, requiring the focusing to be achieved by making the external lens surface concave. As the market is suspicious of concave front curvature for patient contact, the acoustic stack can be curved to match a convex interior lens surface, while keeping the lens front flat. Curving the stack has manufacturing disadvantages and is sometimes prohibitive to maintaining element integrity across the entire array.

To obtain a flat or convex surface without curving the stack, a compound lens of two or more different materials can be used [9]-[11]. We have earlier presented a compound diverging add-on lens for an RCA probe which obtained a FOV of $31^{\circ}$ [12]. The objective of this work is to integrate the developed compound lens into a fully functioning RCA probe and to compare it to a similar probe without a diverging lens. 


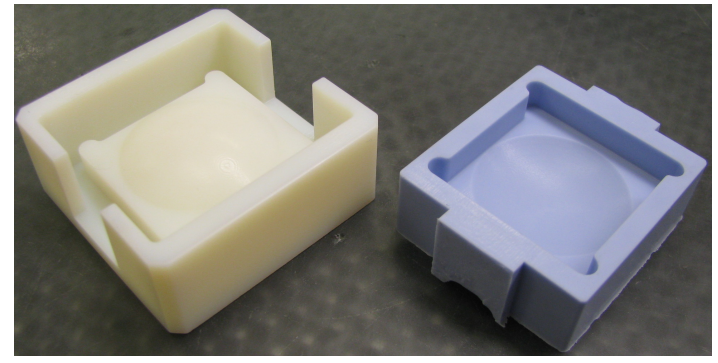

Fig. 1. The inverse mold of the inner lens is 3D printed and bead blasted (left) and a mold is cast of RTV (right).

\section{MEthOD}

\section{A. RCA array}

Two $92+92$ channels RCA 2D CMUT arrays with a $180 \mu \mathrm{m}$ element pitch were fabricated using a LOCOS process similar to the array described in [13], [14].

\section{B. Probe Assembly}

The development of the compound lens, including the composite materials, are described in [12].

The first step of the assembly was to form the inner lens. The inverse of the mold for casting the lens was 3D printed and bead-blasted. The bead-blasting smooths any irregularities originating from the 3D print and textures the surface to obtain a better adhesion. RTV664 was cast in the 3D printed mold and cured overnight at $45^{\circ} \mathrm{C}$. RTV664 was used as the mold material, as silicones and urethanes do not adhere to each other and are commonly used in the industry for molds for the opposite material. The 3D printed inverse mold and the RTV mold are shown in Fig. 1.

The array was cleaned with IPA, plasma ashed, primed with Nusil MED1-161, blown dry with a nitrogen gun, and left in a humidity chamber for 1 hour. The array was then primed with Hapco primer 810 and blown dry with a nitrogen gun. The second primer only requires to set for a couple of minutes. Hapflex 541 was mixed in a ratio of 2:1 (A:B) in a centrifugal mixer for 2 minutes at $2000 \mathrm{rpm}$. Hapflex 541 was then directly poured into the mold and degassed for 2 minutes. The array was then pushed into the self-aligning mold and a load was placed in the backside of the array to push it completely into the mold. Hapflex 541 was then cured overnight at room temperature in a nitrogen atmosphere at $375 \mathrm{kPa}$ to help reduce voids as suggested by the manufacturer. The array was then removed from the mold and post-cured for 6 hours at $45^{\circ} \mathrm{C}$. The array with the inner lens is shown in Fig. 2 .

The array and the inner lens were glued to the nose piece with precision to obtain the required lens thickness as shown in Fig. 3.

A mold of RTV664 was cast around a dummy nose piece where Teflon tape was applied on its surface to avoid the RTV adhering to the nose piece and also to make the cavity in the mold a little larger than the nose piece itself. RTV615 $+\mathrm{Bi}_{2} \mathrm{O}_{3}$ was mixed, in a mass mix ratio (MMR) of 1:0.9, as described in [12]. The RTV664 mold was then placed on a glass plate,
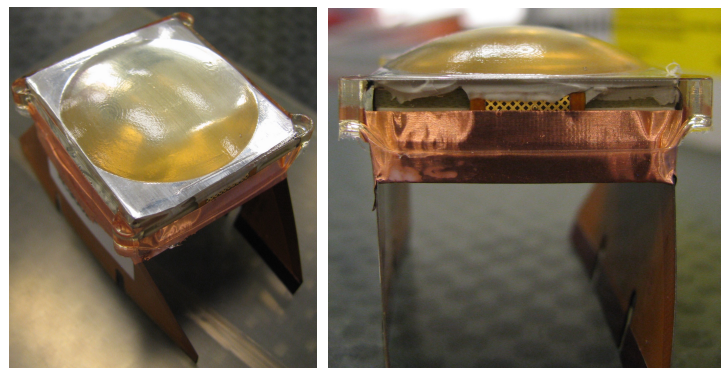

Fig. 2. The mold is filled with Hapflex 541, and after degassing the array is pushed down into it. The Hapflex is cured overnight at $375 \mathrm{kPa}$ at room temperature.

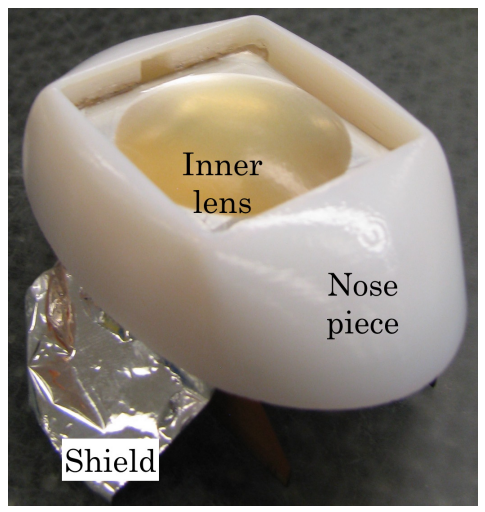

Fig. 3. The transducer with the inner lens was placed in the $3 \mathrm{D}$ printed nose piece and the setback was performed to obtained the required lens thickness.

filled with the RTV615 $+\mathrm{Bi}_{2} \mathrm{O}_{3}$ mixture, and degassed for three minutes. The array and nose piece were then pushed into the mold and pressed against a glass plate to obtain a flat front surface. The assembly was then placed in a spring loaded holder to hold the pieces together during curing. The outer lens was cured overnight at $45^{\circ} \mathrm{C}$. The final nose piece connected to the electronics is shown in Fig. 4.

\section{Characterization}

The transmit impulse responses of the rows of both probes were measured using an AIMS III intensity measurement

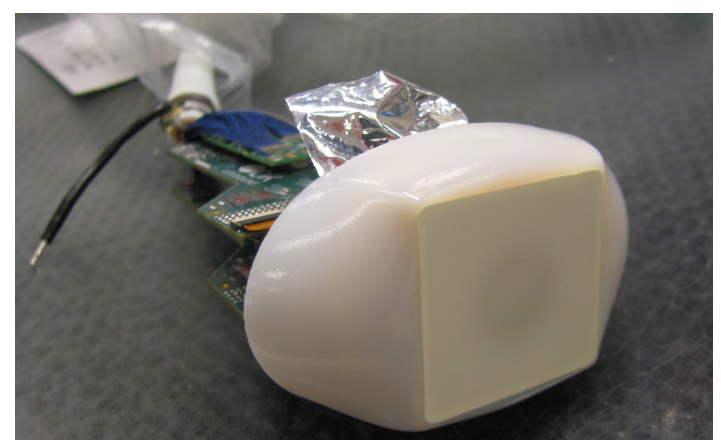

Fig. 4. The nose piece connected to the electronics after the outer lens had cured. The metal sheet sticking out of the nose piece is the electromagnetic shield. 
system (Onda Corp., California, USA) with an Onda HGL0400 hydrophone connected to the experimental research ultrasound scanner, SARUS [15]. The method used to estimate the transmit impulse response is described in [16].

The average impulse response of both probes in the time domain are shown in Fig. 5 and in the frequency domain in Fig. 6(a). The lens is seen to decrease the amplitude of the signal with a factor of six. This effect is also observed in the frequency response. Here the peak amplitude is $10.7 \mathrm{~dB}$ lower and the difference increases with the frequency as shown in Fig. 6(b). A linear fit of the difference between the two frequency responses resulted in a slope of $-4 \mathrm{~dB} / \mathrm{MHz}$, which is caused by the increased attenuation of the lens. The estimate of the one-way attenuation at $5 \mathrm{MHz}$ at the center and at the edge of the lens is $9.9 \mathrm{~dB}$ and $18.9 \mathrm{~dB}$, respectively [12]. Whereas the estimate of the one-way attenuation of the acoustic window of the probe without the lens is $3.2 \mathrm{~dB}$ at $5 \mathrm{MHz}$. This is consistent with the $10 \mathrm{~dB}$ difference at $5 \mathrm{MHz}$ between the two probes.

The center frequency of the impulse response can be calculated as a weighted mean of the frequencies present in the received signal, $S$, as:

$$
f_{c}=\frac{\sum_{i=0}^{N / 2} S\left(i f_{s} / N\right) \cdot i f_{s} / N}{\sum_{i=0}^{N / 2} S\left(i f_{s} / N\right)},
$$

where $N$ is the number of frequency bins in the two-sided spectrum and $f_{s}$ is the sampling frequency. The center frequency decreased from $8.5 \mathrm{MHz}$ to $4.9 \mathrm{MHz}$ by applying the lens. The lower center frequency is also observed in the time domain as the pulse length is longer with the lens applied. These effects are caused by the attenuation of the lens. The attenuation increases with frequency, therefore the high frequency components are attenuated more. This lowers the center frequency and decreases the overall signal amplitude. The $-6 \mathrm{~dB}$ bandwidths decreased from $9.5 \mathrm{MHz}$ to $5.0 \mathrm{MHz}$ by applying the lens. This corresponds to the relative bandwidth decreased with seven percentage points from $109 \%$ to $102 \%$.

The desired effect of the lens was to diverge the acoustic energy. This corresponds to applying a time delay across the transducer. The time delay of the impulse responses across the row elements relative to the average impulse response is shown in Fig. 7 for both probes. The diverging effect is visible, as signals are delayed more at the edges than at the center. The $\mathrm{f}$ number, $F_{\#}$, of the lens can be estimated from the time delay profile. This is done by multiplying the time delay with the speed of sound to obtain the delay "distance". The radius is estimated by fitting a circle to the delay profile and dividing the radius with the width of the active footprint of the transducer. By doing so, the f-number was estimated to $F_{\#}=1.97$. This corresponds to a FOV of $28.5^{\circ}$, while it was designed to $30.1^{\circ}$. A $1.6^{\circ}$ lower FOV could be due to that the probe was a little deformed during curing of the last lens material, which was caused by the spring loaded holder. This could have changed the lens from being flat, to being slightly curved.

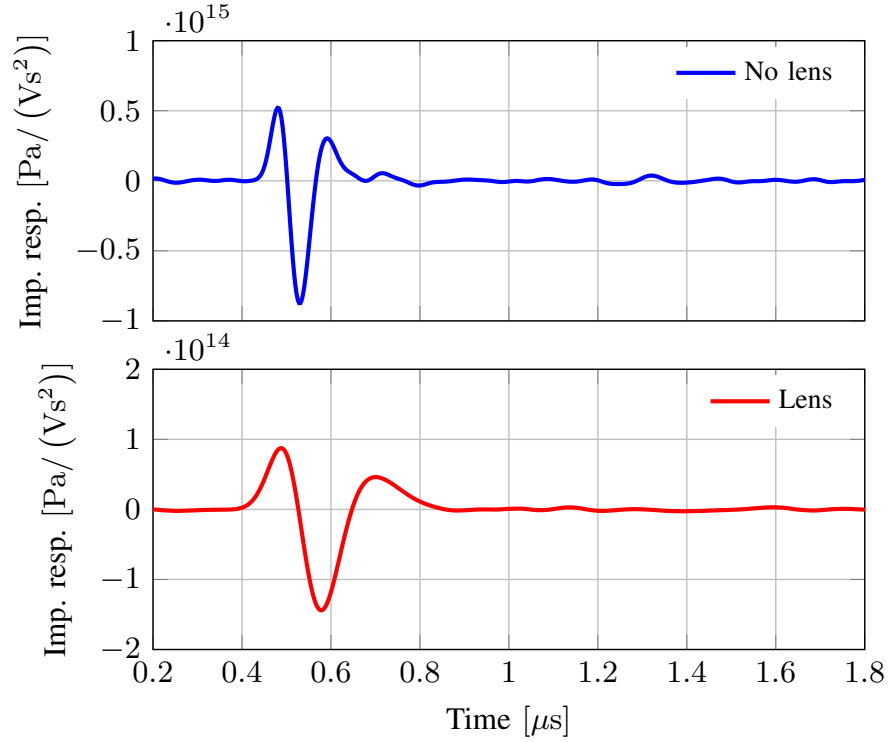

Fig. 5. Time transmit average impulse response of the two probes. The method used to estimate the transmit impulse response is described in [16].

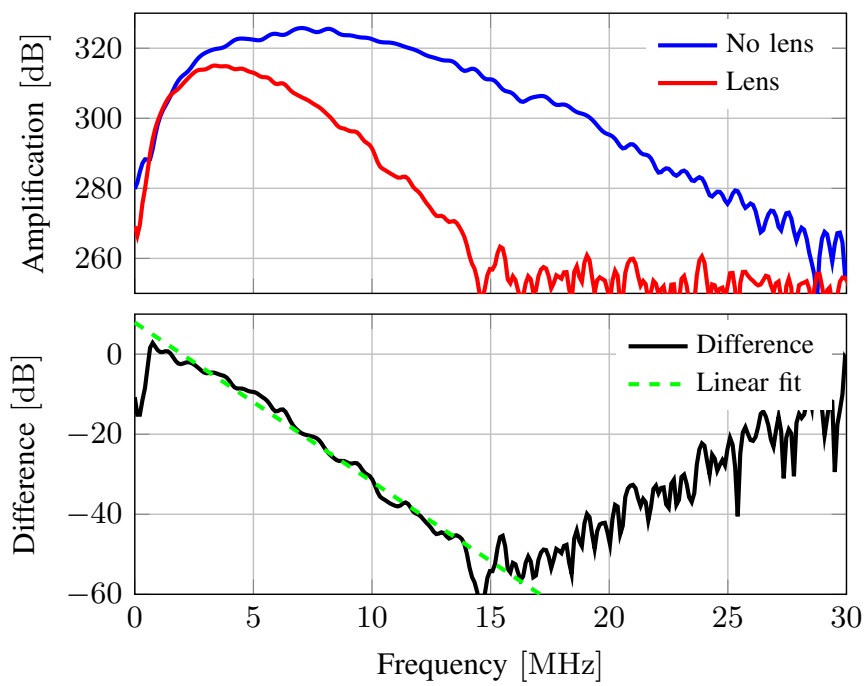

Fig. 6. Frequency impulse response derived from the time impulse response in Fig. 5. A linear fit of the difference between the two frequency responses results in a slope of $-4 \mathrm{~dB} / \mathrm{MHz}$

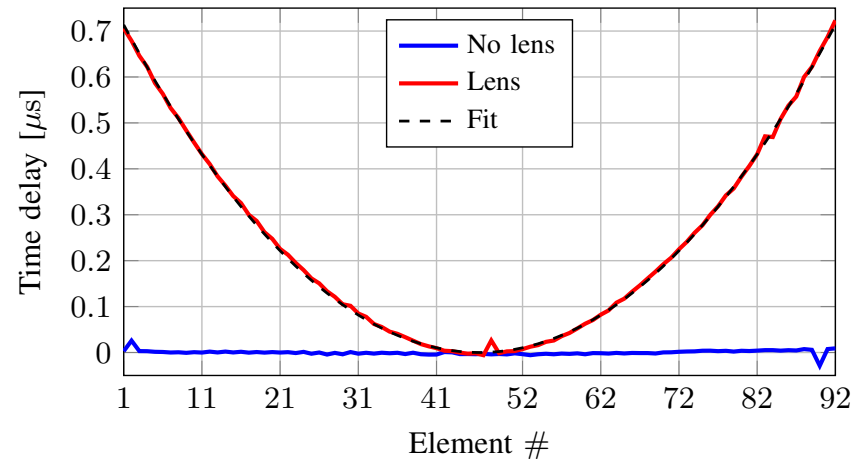

Fig. 7. Comparison of the measured time delay of the row elements of the two probes. A flat time delay profile are seen for the probe without the diverging lens, where the probe with the diverging lens has parabolic formed time delay profile. The f-number was estimated to $F_{\#}=1.97$, corresponding to a FOV of $28.5^{\circ}$. 


\section{CONCLUSion}

Diverging lenses showed to be a feasible method for improving the FOV of RCA arrays. However, common lens materials have a speed of sound lower than tissue/water. The form factor of the lens therefore has to be concave to diverge the energy. A concave front makes patient contact difficult as air can be trapped between the patient and the transducer. A compound diverging lens was integrated into a fully functioning RCA probe to demonstrate the manufacturing capability. A curvilinear FOV of $28.5^{\circ}$ was obtained by applying the lens.

The probe with the diverging lens was compared to a similar probe without a lens. The output pressure was a factor of 6 lower and the center frequency decreased from $8.5 \mathrm{MHz}$ to $4.9 \mathrm{MHz}$ by applying the lens. This was caused by the thicker lens thickness, resulting in an increased attenuation of the transmitted signal.

Compound lenses are challenged due to minimum constituent material thickness requirements and the acoustic attenuation of the materials. A compound lens will always be thicker than then the corresponding single materials lens. Alternatives to cylindrical/spherical shapes are therefore attractive to reduce the total lens thickness. However, RCA arrays might be less sensitive to the high attenuation through the lens as the element size (and therefore transmitted or received energy) is more than twice the size of $1 \mathrm{D}$ arrays.

\section{ACKNOWLEDGMENT}

This work is financially supported by the Danish National Advanced Technology Foundation (82-2012-4), Innovation Fund Denmark (7050-00004B), and by BK Medical, Herlev, Denmark.

\section{REFERENCES}

[1] C. E. Morton and G. R. Lockwood, "Theoretical assessment of a crossed electrode 2-D array for 3-D imaging," in Proc. IEEE Ultrason. Symp., 2003, pp. 968-971.

[2] C. H. Seo and J. T. Yen, "A 256 x 256 2-D array transducer with rowcolumn addressing for 3-D rectilinear imaging," IEEE Trans. Ultrason., Ferroelec., Freq. Contr., vol. 56, no. 4, pp. 837-847, April 2009.

[3] A. Sampaleanu, P. Zhang, A. Kshirsagar, W. Moussa, and R. Zemp, "Top-orthogonal-to-bottom-electrode (TOBE) CMUT arrays for 3-D ultrasound imaging." IEEE Trans. Ultrason., Ferroelec., Freq. Contr., vol. 61, no. 2, pp. 266-276, 2014.

[4] M. F. Rasmussen, T. L. Christiansen, E. V. Thomsen, and J. A. Jensen, "3-D imaging using row-column-addressed arrays with integrated apodization - Part I: Apodization design and line element beamforming," IEEE Trans. Ultrason., Ferroelec., Freq. Contr., vol. 62, no. 5, pp. 947-958, 2015.

[5] A. W. Joyce and G. R. Lockwood, "Crossed-array transducer for realtime 3D imaging," in Proc. IEEE Ultrason. Symp., 2014, pp. 2116-2120.

[6] H. Bouzari, M. Engholm, C. Beers, M. B. Stuart, S. I. Nikolov, E. V. Thomsen, and J. A. Jensen, "Curvilinear 3-D imaging using row-columnaddressed 2-D arrays with a diverging lens: Feasibility study," IEEE Trans. Ultrason., Ferroelec., Freq. Contr., vol. 64, no. 6, pp. 978-988, 2017.

[7] H. Bouzari, M. Engholm, C. Beers, S. I. Nikolov, M. B. Stuart, E. V. Thomsen, and J. A. Jensen, "Curvilinear 3-D imaging using row-column addressed 2-D arrays with a diverging lens: Phantom study," IEEE Trans. Ultrason., Ferroelec., Freq. Contr., vol. 65, no. 7, pp. 1182-1192, 2018.

[8] S. Yang, W. Qin, H. Guo, T. Jin, N. Huang, M. He, and L. Xi, "Design and evaluation of a compound acoustic lens for photoacoustic computed tomography," Biomed. Opt. Express, vol. 8, no. 5, pp. 2756-2765, May 2017.
[9] D. G. Miller, "Transducer acoustic lens," June 1983, US Patent $4,387,720$

[10] J. E. Snyder, L. J. Keres, and G. W. Frey, "Compound lens for ultrasound transducer probe," Nov 1996, US Patent 5,577,507.

[11] D. R. Dietz, C. G. Oakley, D. Morgan, and D. Patwa, "Apodizing ultrasonic lens," February 2011, US Patent 7,888,847 B2.

[12] M. Engholm, H. Bouzari, C. Beers, J. A. Jensen, and E. V. Thomsen, "Increasing the field-of-view of row-column-addressed ultrasound transducers: implementation of a diverging compound lens," Ultrasonics, vol. 88, pp. 97-105, 2018.

[13] M. Engholm, T. L. Christiansen, C. Beers, J. P. Bagge, L. N. Moesner, H. Bouzari, A. Lei, M. Berkheimer, M. B. Stuart, J. A. Jensen, and E. V. Thomsen, "A hand-held row-column addressed CMUT probe with integrated electronics for volumetric imaging," in Proc. IEEE Ultrason. Symp., 2015, pp. 1-4.

[14] M. Engholm, H. Bouzari, T. L. Christiansen, C. Beers, J. P. Bagge, L. N. Moesner, S. E. Diederichsen, M. B. Stuart, J. A. Jensen, and E. V. Thomsen, "Probe development of CMUT and PZT row-columnaddressed 2-D arrays," Sens. Actuators A: Phys., vol. 273, pp. 121-133, 2018.

[15] J. A. Jensen, H. Holten-Lund, R. T. Nilsson, M. Hansen, U. D. Larsen, R. P. Domsten, B. G. Tomov, M. B. Stuart, S. I. Nikolov, M. J. Pihl, Y. Du, J. H. Rasmussen, and M. F. Rasmussen, "SARUS: A synthetic aperture real-time ultrasound system," IEEE Trans. Ultrason., Ferroelec., Freq. Contr., vol. 60, no. 9, pp. 1838-1852, 2013.

[16] J. A. Jensen, "Safety assessment of advanced imaging sequences, II: Simulations," IEEE Trans. Ultrason., Ferroelec., Freq. Contr., vol. 63 , no. 1, pp. 120-127, 2016. 